\title{
Effects of Psychiatric Consultation on Medical Consumption in Medical Outpatients with Low Back Pain*
}

\author{
Gerrit T. Koopmans, M.D., Ludwien Meeuwesen, Frits J. Huyse, and \\ Jan J. Heimans
}

\begin{abstract}
A randomized, controlled, clinical trial $(\mathrm{N}=104)$ was conducted to test the hypothesis that a protocol of collaboration and communication between neurologist and general practitioner, sustained with psychiatric consultation, would reduce medical consumption (especially of diagnostic procedures and medication) in medical outpatients with low back pain. The interoention was designed at the health care provider level; the psychiatrist did not see or examine the patient. A reduction in medical consumption could not be demonstrated. As there was a great variation in adherence to the protocol in the experimental group, this might explain the lack of hypothesized effects. Full implementation of the protocol seemed to imply a lower number of major surgery operations, but this effect disappeared after excluding cases with a diagnosed hernia. The possibilities for consumption reduction for three post hoc-defined patient categories (cases with hernia and chronic and nonchronic cases) and the consequences for patient selection and treatment intensity are discussed. It is concluded that the target groups
\end{abstract}

*This study was conducted as part of a larger on-going project entitled "Interaction Between Primary and Secondary Health Care" (Academic Hospital of the Free University, Amsterdam, the Netherlands). Participating members of the research group include Y. Dolstra, GP; Professor A.J.M. Donker, internist; Professor R. van Dyck, psychiatrist; J.J. Heimans, neurologist; F.J. Huyse, psychiatrist; L. Meeuwesen, psychologist; F..M. Meiland, psychologist; J.G. Streefkerk, GP; and D. Wijkel, medical sociologist.

Department of Health Care Policy and Management, Erasmus University, Rotterdam, The Netherlands, (G.T.K.) Research Center Primary/Secondary Health Care (L.M.), Departments of Consultation-liaison Psychiatric Service (F.J.H.), and Neurology (J.J.H.) the Free University Hospital, Amsterdam, The Netherlands

Address reprint requests to: Gerrit T. Koopmans, Department of Health Care Policy and Management, Erasmus University, P.O. Box 1738, 3000 DR Rotterdam, The Netherlands. should be more narrowly defined, and that a more intensive intervention might prove to be more effective.

\section{Introduction}

There is considerable evidence for the existence of psychological comorbidity among a relatively large proportion of medical patients who are consulting a general practitioner (GP) or a medical specialist in an outpatient clinic, or are being treated in a general hospital [1-6].

Low back pain (LBP) belongs to the category of somatic complaints which are often associated with psychological comorbidity [7]. Moreover, LBP is a common phenomenon in the general population [8], and one of the most important reasons for consulting a physician $[2,9,10]$.

Back pain has a substantial effect on health care utilization and costs [11]. Its costs in 1990 in the US were estimated to be more than $\$ 24$ billion, and the total (including indirect) costs might rise into the $\$ 75-\$ 100$ billion range [8], mostly because of the relatively long average duration (23-30 days) of lost work episodes [12]. In the Netherlands, direct hospital costs (in 1988) for treating back pain patients were almost Dfl. 230 million ( 1 Dfl $=0.50$ US $\$$ ), in a population of 15 million [13]. Almost $16 \%$ of the yearly incidence of disability in the Netherlands is attributable to diagnosis related to back pain [14].

Low back pain poses a problem not only because of its consequences, but also because of its complicated etiology. The classical pathology model is not very helpful in many cases of low back pain. In a large percentage of symptomatic patients, no struc- 
tural lesions can be found, although asymptomatic individuals can show structural abnormalities in the lumbar spine [15]. Only $10 \%-20 \%$ of patients with acute LBP can be given a precise pathoanatomical diagnosis; in most cases, diagnoses are unspecific [16]. The complexity of diagnosing LBP is illustrated by the fact that the average costs of establishing an organic diagnosis in these cases were found to be high, (\$7263) [7].

Although the nature of the association of LBP and emotional distress is ambiguous [17-19], there seems to be common ground for the observation that in the treatment of LBP the high incidence of psychological comorbidity cannot be ignored. In medical populations the relationship between psychopathology and an increase of health service utilization, and the impact of psychological interventions, have been extensively described $[20,21]$. As for LBP patients, there is evidence that the same kind of relationship exists [19,22-24].

Consequently, a disease concept, which dismisses the dichotomy of organic vs psychogenic (functional) disorder in LBP, is gaining support $[15,24]$. An integrated disease concept, such as the biopsychosocial concept, seems to provide for a better understanding of these complaints [17]. Intervention models based on this concept might lead to a better response to treatment. Consultation liaison psychiatry has developed and tested these models in several medical and surgical (outpatient) populations [25-27].

This study applies the principles of a biopsychosocial model in a randomized controlled study in patients with LBP referred to an outpatient neurology clinic by their GP, in order to assess its effects on their health service utilization. Consequently, a randomized controlled experiment was conducted in the outpatient clinic of the neurology department of the Free University Hospital in Amsterdam.

It was hypothesized that the implementation of standard guidelines for communication and collaboration by medical specialist and GPs, including an integrated approach to diagnosis and treatment, would change the utilization patterns of these patients. The collaboration and communication between the referring GP and medical specialist are of special significance in the Dutch health care system, which has a two-tiered approach for screening and treatment [28]. Ideally, the first tier is the domain of the GP; the second tier medical specialists are only consulted on referral by a GP. However, in cases of referral, the communication between these two is restricted.

\section{Method}

Subjects

All patients referred by a GP to the outpatient clinic of neurology with complaints of LBP during a period of 12 months, beginning August 1989, were eligible for the study. Target complaints included LBP with or without irradiating pain in one or both legs. Exclusion criteria included age outside the range of 18-65 years, illiteracy or poor ability to manage the Dutch language, or the presence of evident somatic pathology (e.g., cancer or other malignancies, as well as back pain caused by hip affections or referred pain). New consecutive attenders meeting these criteria were requested to volunteer for participation in the study. Their second visit included an informed consent procedure (Fig. 1).

\section{Randomization}

Medical specialists participating in the study were uniquely allocated to either the experimental or the control condition. During the experiment, one group of four neurologists participated in the experimental condition, and another group of four neurologists participated in the control condition. Both groups were working in their own subdepartments within the outpatient clinic, providing care independent from each other, in order to minimize carry-over effects. Patients were randomly distributed over both conditions, i.e., groups of specialists, resulting in, 50 patients in the experimental condition and 54 in the control condition.

\section{Intervention}

The primary focus of the intervention was the enhancement of communication between neurologists and general practitioners (GPs) through two standardized telephone calls to patients at risk for somatization, and a screening by a research assistant for somatization risk factors which included a history of high health service utilization and the presence of psychological distress.

After enrollment in the study, there was a first integral assessment of the patient by the research assistant (for a more extensive description of the instruments, see Meeuwesen et al. [29]. In the meantime there was telephone communication between neurologist and GP, focusing on the verification of the data provided by the patient, and checking the reason for referral. A second telephone com- 


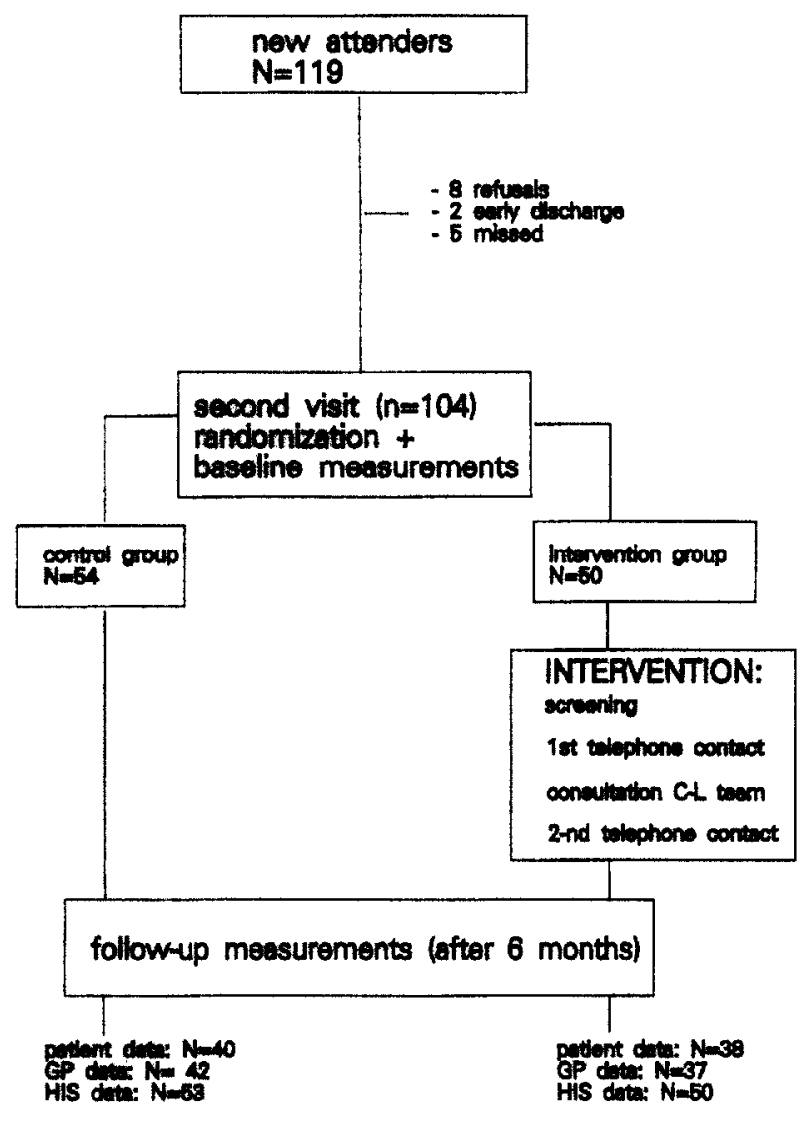

Figure 1. Study design.

munication took place between the neurologist and the GP to agree upon an intervention program for the patient based on the results of the information provided by the GP and the physical and psychological findings. In between these two telephone calls, during a supervision session, the psychiatrist reviewed the findings from the physical assessment with the neurologist, the additional psychosocial assessment, including the scales, and those findings obtained through communication by phone with the GP. During the review of the findings, the psychiatrist, neurologist, and the research assistant decided whether or not physical and/or psychological factors played an important role in the current state, and whether or not the organization of the patient's health care was functional. On the basis of the formulated conclusions, a treatment plan was recommended, which was selected from a menu of operationalized interventions [30]. The main possibilities were 1) referral back to the GP (autonomous care by GP), 2) joint care (GP and neurologist), 3) autonomous care by neurologist or psychiatrist, or 4) referral to mental health care institutions. This plan was communicated by the neurologist to the GP by a second telephone call, in which the neurologist and the primary care physician designed a definite care plan program for the patient, which was discussed with the patient by the neurologist. In the case of a 'high risk' patient, a division of tasks was also discussed, this included reassuring the patient, communicating the coexistence of psychological symptoms, and if necessary, referral to mental heath care.

In this experiment the main role of the psychiatrist in the intervention was the implementation of this standard assessment and communication program and the interpretation of the collected results. The psychiatrist did not see the patient her or himself.

\section{Hypotheses}

It was expected that this intervention would cause 1) a more effective and satisfying collaboration and communication pattern between GPs and medical specialists; 2) an improvement (or at least not a worsening) of the physical and psychological status of the patients; 3 ) a more effective and less costly pattern of health care for these patients, i.e., more care provided by the GP, fewer prescriptions of medicines, fewer diagnostic procedures, and less usage of paramedic services and those of nontraditional providers.

\section{Assessment}

At baseline the following assessments were made:

A Somatization subscale derived from DSM-III-R [31,32]

The 28-item version of the General Health Questionnaire (GHQ) [33,34]

Four dimensions of the revised (Dutch) version of the Symptom Checklist (SCL)-90 [35]: anxiety, depression, somatization, and psychoneuroticism, using norms developed for the target population [36]

A Functional Status subscale of the Quality-of-Life Assessment [37].

At follow-up 6 months after the date of randomization, all patients were interviewed and reassessed with the same instruments used for baseline measurements. Medical consumption in the past 6 months was measured by using three different sources: patients, GPs of the referred patients, and the Hospital Information System (HIS). Using these 
data, several indicators for medical consumption were constructed according to the categories that were expected to differentiate between the intervention and the control group. Aggregation of indicators over data sources was not considered due to the usage of different measurement formats, nonsystematic missing data, and differences in scope of each source. The use of different sources for analogous consumption indicators allows one to judge the generalizability of the results.

Most indicators are based on counts of events of medical consumption within a specified category, as registered by the different sources. Indicators based on data from the Hospital Information System were also partly translated in monetary terms. Since information about real costs was not available, each service unit was given a 'price-tag' calculated as a weighted mean of the two different fees that are used for the same service unit: one for sickness fund patients and one for privately insured patients, which were weighted $60 \%$ and $40 \%$, respectively, in accordance with their population proportion.

\section{Data Analyses}

To test the hypotheses concerning differences in medical consumption between intervention and control groups, and because the distribution of variables of medical consumption tends to be skewed, nonparametric tests (Mann Whitney U test, Kruskal Wallis test) were used.

\section{Results}

\section{Sample Characteristics}

There were no significant differences in sociodemographic profile between experimental and control groups (see Table 1). Both groups are comparable in the distribution of age, sex, educational and professional level; marital status, and type of health insurance.

Response rates at follow up were between $75 \%$ (patient data) and 76\% (GP data). In the experimental condition these figures were $76 \%$ and $74 \%$, respectively, and in the control condition $74 \%$ and $78 \%$, respectively.

There were no differences between respondents and nonrespondents on sociodemographic factors and baseline measures.

\section{Baseline Measures}

Mean scores of measures on psychological and physical well being, taken at baseline, were mostly the same except for significantly higher mean scores on the SCL depression and psychoneuroticism in the control group compared with the experimental group (2-sided $t$-tests, $p<0.05$ ).

\section{Intervention Effects on Psychological and Physical Well-being}

In general, comparing baseline and follow-up measures in both groups, patients showed a significant improvement on the Functional Status index (intervention group: $p=0.00$; control group: $p=0.04$ ) and a tendential significant improvement on the general feeling of health (as measured with the GHQ-28; intervention group: $p=0.07$; control group: $p=$ 0.12 ). Although there was a significant two-way (condition * time) multivariate interaction effect, a differential effect of the experimental and control condition on the extent of improvement on any specific measure used could not be established. A more comprehensive description of the clinical effects of the intervention can be found in Meeuwesen et al. [29].

\section{Intervention Effects on Collaboration and Communication Between Physicians}

In contrast to the hypothesis, no differences in satisfaction concerning the treatment plan, collaboration, and communication were found between GPs of patients in the intervention group and their colleagues in the control group. In the letters of the specialists in the intervention group, more conclusions regarding psychopathology were found [29].

\section{Intervention Effects on Medical Consumption}

Diagnostics. Table 2 gives an overview of several key indicators for the utilization of diagnostic procedures (such as lab tests, X-rays, CT scans). No differences were found between both groups in the utilization of diagnostic procedures initiated by the GP. However, specialists tended to initiate more diagnostic procedures for patients in the experimental condition (according to HIS data: $\mathrm{z}=-2,34 ; p=$ $0.02)$, which is also reflected in the costs of such procedures $(z=2.34 ; p=0.02)$. This difference was not confirmed, however, by the patient data $(\mathrm{z}=$ $-0.91 ; p=0.36$ ). 
In summary, it can be stated that the expected lower utilization of diagnostic procedures in the intervention group, especially those initiated by the neurologists and other medical specialists, could not be confirmed. On the contrary: evidence appears to point more towards an adverse effect.

Medication. Patients in the experimental condition did not receive fewer prescriptions for medicines, as was expected (see Table 3). GPs seemed to prescribe less medicine to patients in the intervention group, according to patient data, but this difference was not significant. As more psychopharmaca might be prescribed in the experimental condition, a differentiation was made in the type of prescriptions. However, no difference were found. According to the GP data, 17 patients received such prescriptions ( 8 in the intervention group, 9 in the control group), and according to patient data, 5 patients ( 1 in the intervention group, 4 in the control group) received such a prescription from a medical specialist.

Consultations (Table 4). In contrast to the hypothesis, patients in the experimental condition did not visit their GP more frequently (based on GP data: $\mathrm{z}=-0.13 ; p=0.897$; based on patient data: $\mathrm{z}=$ $-0.55 ; p=0.579$ ), nor was there any significant effect on the number of visits to the neurologist was well as to other specialists. For both groups, the number of consultations of paramedic professionals and nontraditional providers was more or less the same. Both groups consulted a physiotherapist quite often, but patients from the intervention group did not do so more often than patients from the control group.

Major and Minor Surgery (Table 5). The number of minor surgical procedures (not requiring hospitalization) did not differ significantly between both groups. Although the mean costs for these procedures in the intervention group was double those of the control group, this was not a significant difference.

The number of major surgical procedures (i.e., requiring hospitalization) was higher in both groups than the number of minor surgical procedures. In the intervention group, 10 patients received one operation and one patient two, whereas in the control group, 8 patients received one operation. This difference is reflected in the mean costs. However, both differences did not reach the level of significance.
Table 1. Demographic profile of intervention and control groups

\begin{tabular}{|c|c|c|c|c|c|c|}
\hline & \multicolumn{2}{|c|}{ Intervention } & \multicolumn{2}{|c|}{ Control } & \multicolumn{2}{|c|}{ Total } \\
\hline & $N$ & $\%$ & $N$ & $g$ & $N$ & $\%$ \\
\hline \multicolumn{7}{|l|}{ Age (years) } \\
\hline $18-29$ & 7 & 14 & 11 & 20 & 18 & 17 \\
\hline $30-39$ & 17 & 34 & 11 & 20 & 28 & 27 \\
\hline $40-49$ & 13 & 26 & 14 & 26 & 27 & 26 \\
\hline $50-59$ & 11 & 22 & 13 & 24 & 24 & 23 \\
\hline $60-65$ & 2 & 4 & 5 & 9 & 7 & 7 \\
\hline \multicolumn{7}{|l|}{ Sex } \\
\hline Male & 21 & 42 & 16 & 30 & 37 & 36 \\
\hline Female & 29 & 58 & 38 & 70 & 67 & 64 \\
\hline \multicolumn{7}{|l|}{ Education } \\
\hline Low & 25 & 50 & 19 & 35 & 44 & 42 \\
\hline Medium & 10 & 20 & 17 & 32 & 27 & 26 \\
\hline High & 15 & 30 & 18 & 33 & 33 & 32 \\
\hline \multicolumn{7}{|l|}{ Occupation } \\
\hline Low & 18 & 36 & 11 & 20 & 29 & 28 \\
\hline Medium & 17 & 34 & 20 & 37 & 37 & 36 \\
\hline High & 8 & 16 & 10 & 19 & 18 & 17 \\
\hline No occup. & 7 & 14 & 13 & 24 & 20 & 19 \\
\hline \multicolumn{7}{|l|}{ Marital status } \\
\hline Unmarried & 7 & 14 & 10 & 19 & 17 & 16 \\
\hline $\begin{array}{l}\text { Married/Long- } \\
\text { lasting intimate }\end{array}$ & & & & & & \\
\hline relationship & 37 & 74 & 40 & 74 & 77 & 74 \\
\hline \multicolumn{7}{|l|}{ Divorced/ } \\
\hline separated & 2 & 4 & 3 & 0 & 5 & 5 \\
\hline Widowed & 4 & 8 & 1 & 2 & 5 & 5 \\
\hline \multicolumn{7}{|l|}{$\begin{array}{l}\text { Health insurance } \\
\text { status }\end{array}$} \\
\hline Sickness fund & 34 & 68 & 39 & 72 & 73 & 70 \\
\hline Private & 16 & 32 & 15 & 28 & 31 & 30 \\
\hline \multicolumn{7}{|l|}{ Ethnicity } \\
\hline NW European & 48 & 96 & 50 & 93 & 98 & 94 \\
\hline Other & 2 & 4 & 4 & $?$ & 6 & 6 \\
\hline
\end{tabular}

Hospital Days (Table 5). Twenty-three patients from the intervention group were admitted into a hospital at least once ( 6 twice, 1 three times); in the control group, 21 patients were admitted at least once ( 3 twice, 1 three times), a nonsignificant difference. For length of stay, the differences tend to point in the same direction, i.e., in the intervention group the mean length of stay was about 2 days higher compared with the control group, also a nonsignificant difference.

\section{Discussion}

Contrary to expectations, the intervention did not effectuate a reduction of medical consumption. As 
Table 2. Utilization of diagnostic procedures by intervention and control groups during a 6-month period

\begin{tabular}{|c|c|c|c|c|c|c|c|c|}
\hline & \multicolumn{3}{|c|}{ Intervention } & \multicolumn{3}{|c|}{ Control } & \multicolumn{2}{|c|}{ Analysis } \\
\hline & $N$ & Mean & SD & $N$ & Mean & $\mathrm{SD}$ & $z$ & $p$ \\
\hline Number of procedures initiated by $\mathrm{GP}^{a}$ & 38 & 1.16 & 2.72 & 40 & 0.85 & 1.37 & -0.23 & 0.817 \\
\hline Number of procedures initiated by $\mathrm{GP}^{h}$ & 37 & 0.59 & 0.83 & 42 & 0.52 & 0.80 & -0.35 & 0.727 \\
\hline Number of proc & 38 & 1.16 & 2.20 & 40 & 1.20 & 1.52 & -0.91 & 0.364 \\
\hline Number of procedures initiated by specialist ${ }^{c}$ & 50 & 1.44 & 1.49 & 53 & 0.98 & 1.49 & -2.34 & 0.020 \\
\hline Costs (in Df) of procedures initiated by specialist ${ }^{c}$ & 50 & 200 & 281 & 53 & 134 & 245 & -2.34 & 0.019 \\
\hline
\end{tabular}

${ }^{a}$ According to patient.

${ }^{b}$ According to GP.

${ }^{c}$ According to HIS.

far as any effect on medical consumption could be demonstrated, it was the reverse.

As the intervention and control group were unbalanced at baseline on two measures of psychological distress (SCL-depression and psychoneuroticism), we decided to perform some additional analyses controlling for these factors. The results of the regression analyses, using log-transformed scores of the outcome measures, confirmed the results of the analysis as reported in the previous section.

As a general explanation of these negative findings, the possibility has to be considered that the study was underpowered due to relatively small size. For small effect sizes $(d=0.2)$ the power of the analyses used is around 20\%. For medium effect sizes $(d=0.5)$ the power of the analyses reported in the results section is about $70 \%$, and for large effect sizes $(d=1.0)$ the power becomes $100 \%$. Since a small effect size would mean that only $1 \%$ of the variance of the dependent variables can be attributed to the intervention [38], we tend to consider effects of this magnitude as irrelevant. For medium effect sizes the power of the analyses is satisfactory. So it can be concluded that the study was not underpowered to detect intervention effects of any substance.

In trying to find some specific explanations for these negative findings, several factors which might have influenced the outcome of the intervention, were explored. We acknowledge the restricted value of these additional analyses as they are subject to statistical limitations. Increasing the number of statistical tests raises the potential of statistical errors of type I. Furthermore, the sample size restricts the power of the analyses, especially when a stratifying variable is introduced. Therefore, these additional analyses are not intended to give ultimate answers, but to generate some more specific hypotheses concerning the (lack of) effectiveness of the intervention.

First we explored the possibility that the intervention failed because it was not implemented as designed. The intervention, which is basically a guideline for collaboration and communication between neurologist and GP under supervision of a CL psychiatrist, is, like any behavioral protocol, vulnerable to disruption due to nonadherence. The second telephone communication, which can be considered essential, took place in $82 \%$ of the cases, according to the project office. However, this was not confirmed in all cases by the GPs. Using both sources of information, three levels of implementation of the intervention (i.e., adherence to the experimental protocol) could be distinguished:

1. Full implementation (second telephone call took place according to the project office and this was confirmed by the GP)

2. Half implementation (second telephone call took place according to the project office, but this was not confirmed by the GP)

3. No implementation (no second telephone call)

The corresponding number of patients were 14, 17, and 5 , respectively (for an additional 14 patients, not enough data were available to make a reasonable choice for the category they belonged to).

Assuming the worst for the 14 patients for whom implementation could not be verified, this means that it is possible that only $28 \%$ of the cases in the experimental condition received the full dosage of the treatment. As such, this is an important obser- 
Table 3. Prescriptions of medicines utilized by intervention and control groups during a 6-month period

\begin{tabular}{|c|c|c|c|c|c|c|c|c|}
\hline & \multicolumn{3}{|c|}{ Intervention } & \multicolumn{3}{|c|}{ Control } & \multicolumn{2}{|c|}{ Analysis } \\
\hline & $N$ & Mean & $\mathrm{SD}$ & $N$ & Mean & SD & $z$ & $p$ \\
\hline Units prescribed by GP ${ }^{a}$ & 38 & 0.58 & 0.68 & 40 & 1.03 & 1.12 & -1.60 & 0.110 \\
\hline Units prescribed by $G P^{b}$ & 37 & 1.11 & 1.37 & 42 & 1.07 & 0,95 & 0.54 & 0.591 \\
\hline Units prescribed by specialist ${ }^{a}$ & 38 & 0.26 & 0.50 & 40 & 0.30 & 0.56 & -0.19 & 0.852 \\
\hline Units of psychopharmacologic agents prescribed by $\mathrm{GP}^{a}$ & 38 & 0.03 & 0.16 & 40 & 0.15 & 0.48 & -1.34 & 0.180 \\
\hline Units of psychopharmacologic agents prescribed by $\mathrm{GP}^{b}$ & 37 & 0.24 & 0.50 & 42 & 0.36 & 0.85 & -0.10 & 0.923 \\
\hline
\end{tabular}

"According to patient.

${ }^{h}$ According to GP.

vation because it might explain the lack of expected outcomes. If it can be shown that the results are in the expected direction, in particularly with patients who received full dosage, the failing implementation may be considered the main cause of our negative findings.

For that reason we compared the three implementation groups that were distinguished (none, half, and full implementation) on the same key indicators used in comparing the intervention and control groups. Just one significant difference was found: the full implementation group had the lowest number of major surgery operations (only 1 out of 14 patients had surgery) and the half implementation group had the highest number ( 8 out of 17 patients were operated on at least once) $\left(\chi^{2}=6,22\right.$; $p=0.045$ ). This difference was also reflected in the costs of major surgery and the number of hospitalizations, although not significantly $\left(\chi^{2}=4,69 ; p=\right.$ 0.096 and $\chi^{2}=2,95 ; p=0.229$ ).

However, this difference might be the result of case mix differences. After the exclusion of cases with hernia, ${ }^{1}$ a reduction of major surgical operations in relation to a full implementation of the guidelines disappeared. In other words, the implementation effect is strongly influenced by the inclusion of cases with hernia, which were found mostly in the half implementation group all of whom received major surgery.

In summary, there was a variation in the adherence to the experimental protocol, which seemed to be related to the number of major surgical opera-

\footnotetext{
${ }^{i}$ In the full implementation group two cases were diagnosed having a hernia (none of which had major surgery), and in the half implementation group 4 cases (all of which had major surgery) and the no implementation group one case (which also had major surgery).
}

tions. After exclusion of cases with hernia, full implementation of the intervention did not prove to make any difference. However, this finding is to be interpreted with caution because of the low number of cases in the groups compared.

As a second option to explain the results, we explored the role of chronicity (a duration of the complaints of at least 6 months), as chronic LBP patients usually show more signs of psychopathology $[18,24]$. Regardless of the experimental condition, major differences were found between chronic $(N=$ 57) and nonchronic $(N=43)$ patients, all in the direction of a higher level of utilization for the nonchronic cases. However, most of these differences disappeared when cases with hernia were excluded, since 13 of the 17 cases with hernia had nonchronic complaints. The remaining differences were not unequivocal: 1) nonchronic patients had a higher number of consultations with the physiotherapist and 2) chronic patients received more drug prescriptions from the GP (according to the GP).

Comparing intervention and control groups within the selection of chronic cases who were not diagnosed as having a hernia, revealed a significantly higher amount of diagnostic procedures in the intervention group $(z=-2.54 ; p=0.01)$. The same comparison within the group of nonchronic, nonhernia cases revealed a higher frequency of consultations of other specialists by the control group $(\mathrm{z}=-2.28 ; p=0.02)$, but also more depressive symptomatology $(z=-2.33 ; p=0.02)$. So the higher number of diagnostic procedures found in general is mainly caused by an adverse effect within the group of chronic cases. Why this is the case, remains to be explained.

A third factor that might influence the effect size 
Table 4. Consultations of providers by intervention and control groups during a 6-month period

\begin{tabular}{|c|c|c|c|c|c|c|c|c|}
\hline \multirow[b]{2}{*}{ Number of Consultations } & \multicolumn{3}{|c|}{ Intervention } & \multicolumn{3}{|c|}{ Control } & \multicolumn{2}{|c|}{ Analysis } \\
\hline & $N$ & Mean & SD & N & Mean & SD & $\mathrm{z}$ & $p$ \\
\hline $\mathrm{GP}^{a}$ & 38 & 2.75 & 2.21 & 40 & 2.63 & 2.36 & -0.55 & 0.579 \\
\hline $\mathrm{GP}^{b}$ & 37 & 2.95 & 0.26 & 42 & 3.07 & 3.00 & -0.13 & 0.897 \\
\hline Neurologist $t^{a}$ & 38 & 2.23 & 2.25 & 40 & 1.70 & 1.42 & -0.51 & 0.614 \\
\hline Neurologist $^{c}$ & 50 & 2.72 & 1.34 & 53 & 2.49 & 1.09 & -0.87 & 0.386 \\
\hline Other specialists $^{a}$ & 38 & 0.45 & 1.08 & 40 & 1.03 & 2.02 & -0.95 & 0.340 \\
\hline Other specialists $^{c}$ & 50 & 0.82 & 1.41 & 53 & 0.58 & 1.13 & -0.73 & 0.464 \\
\hline Physiotherapists ${ }^{a}$ & 38 & 19.45 & 19.67 & 40 & 23.58 & 26.35 & -0.43 & 0.671 \\
\hline Paramedics (excl. physioth.) ${ }^{a}$ & 38 & 1.13 & 4.32 & 40 & 1.63 & 3.84 & -0.33 & 0.744 \\
\hline Nontraditional providers ${ }^{a}$ & 38 & 2.13 & 4.69 & 40 & 2.38 & 6.64 & -1.30 & 0.193 \\
\hline
\end{tabular}

${ }^{a}$ According to patient.

${ }^{b}$ According to GP.

${ }^{c}$ According to HIS.

is the intensity of intervention. The main goal of intervention was to support the GP by securing his pivotal role in the treatment of the patient and reducing the chance of the patient being sucked into the secondary health care system with all its emotional and financial consequences. Yet, as appeared throughout the course of the study, the neurologists themselves took a conservative approach and were inclined to quickly send the patients back to the GP. Even though patients were randomly allocated to the physicians, there might have been a carry-over effect through the attending neurologists as they were aware of the goal to reduce health care utilization in this group of patients. Consequently, this might have been an additional factor reducing the estimated effect size.

As the level of medical consumption and the effects of the intervention (including the probability of adherence) seem to be partly associated with patient characteristics, such as diagnosis and chronicity of the complaints, a distinction in three patient categories is suggested for future intervention studies:

1. Patients who have been diagnosed as having a hernia, suitable for operation. With respect to medical consumption, this category is the most costly, and there is no reason to believe that a protocol as tested in our experiment will reduce these costs significantly, especially when the LBP complaints have a recent onset.

2. Patients with chronic LBP complaints. This cat- egory is often described in the literature as being at risk for psychological disturbances $[18,24]$. In that sense, these patients seem to be suitable for an integrated approach, which might be beneficial to the relief of their complaints. Yet it is questionable, whether such an approach can be justified by expected offset effects. In our experiment, even negative offset effects have been found due to higher utilization of diagnostic procedures. Moreover, chronic patients in our experiment had a comparatively low medical consumption, complicating any experiment focusing on its reduction, and did not show more psychopathology than the nonchronic patients. However, the same protocol might be effective in a different setting where the treatment policy is less conservative.

3. Patients with nonchronic LBP complaints (after exclusion of hernia cases). For these patients, the effectiveness of the experimental protocol to reduce medical consumption is ambiguous. Nonchronic patients in the intervention group consulted other specialists less frequently, but were also less depressed. Since the level of medical consumption is comparable to the chronic patients of this experiment, the same argument holds concerning the possibility of achieving a reduction.

In conclusion, with regard to patient characteristics, our finding point in the direction of more stringent inclusion criteria. First, cases with hernia 
Table 5. Surgery and hospitalization utilized by intervention and control groups during a 6-month period

\begin{tabular}{|c|c|c|c|c|c|c|c|c|}
\hline & \multicolumn{3}{|c|}{ Intervention } & \multicolumn{3}{|c|}{ Control } & \multicolumn{2}{|c|}{ Analysis } \\
\hline & $N$ & Mean & SD & $N$ & Mean & $\mathrm{SD}$ & $z$ & $p$ \\
\hline Number of minor surgery procedures ${ }^{a}$ & 37 & 0.08 & 0.36 & 42 & 0.00 & 0.00 & -1.52 & 0.129 \\
\hline Number of minor surgery procedures ${ }^{b}$ & 50 & 0.08 & 0.40 & 53 & 0.13 & 0.56 & 0.41 & 0.684 \\
\hline Costs (in Dfl) of minor surgery procedures ${ }^{b}$ & 50 & 146 & 721 & 53 & 70 & 491 & 0.35 & 0.730 \\
\hline Number of major surgery procedures ${ }^{b}$ & 47 & 0.26 & 0.49 & 50 & 0.16 & 0.37 & 0.90 & 0.367 \\
\hline Costs (in Dfl) of major surgery procedures ${ }^{b}$ & 50 & 294 & 756 & 54 & 194 & 624 & 0.74 & 0.456 \\
\hline Number of hospitalizations & 50 & 0.62 & 0.78 & 54 & 0.48 & 0.69 & 0.95 & 0.340 \\
\hline Number of hospital-days & 50 & 7.26 & 10.09 & 54 & 5.30 & 10.00 & -1.26 & 0.209 \\
\hline
\end{tabular}

"According to GP.

"According to HIS.

should be excluded. Secondly, we expect that a restriction to cases with more severe psychopathology, when there is also an indication of high utilization of unnecessary medical care, might prove more effective. The fact that no differential effect of the intervention within the group of chronic patients were found, might be the result of the absence of strict inclusion criteria, as the chronic patients did not show more signs of psychopathology than the nonchronic patients, which is usually the case $[18,24]$. If the extent of the care provided to this category is already restricted, a reduction in medical consumption is difficult to observe.

However, restricting a protocol to a more narrowly defined target group might not be enough. The combination of a not very intensive and indirect intervention and a patient group with more severe psychopathology, will probably be ineffective. This possibility exists especially in a situation where normal treatment is already well developed [39], or the complaints are persistent [40]. In accordance with the recommendations of Katon and Gonzales [25], we expect a more intensive intervention-targeting more directly the illness behavior of the patients who run the risk of a redundancy of care-might prove to be more effective. Currently a combination of screening after referral, as described in this study, with a subsequent combined consult in the office of the GP is considered as a possibility to enhance the impact of the current intervention [41].

The authors would like to thank the patients and physicians who took part in this study, Leona van Roijen for summarizing Dutch epide- miological and cost data, Professor F.F.H. Rutten, and the anonymous reviewers for commenting on earlier drafts.

\section{References}

1. Kessler LG, Burns BB, Shapiro S, et al: Psychiatric diagnoses of medical service users: evidence from the Epidemiologic Catchment Area Program. Am J Public Health 77:18-24, 1987

2. Foets $M$, Sixma $H$ : Een nationale studie van ziekte en verrichtingen in de huisartspraktijk. Basisrapport: Gezondheid en gezondheidsgedrag in de praktijkpopulatie (A National Study of Morbidity Patterns in General Practice. Basis Report: Health and Health Behaviour). Utrecht, NIVEL, 1991

3. Ormel J, van den Brink W, Koeter MWJ, et al: Recognition, management and outcome of psychological disorders in primary care: a naturalistic follow-up study. Psychol Med 20:909-923 1990

4. Lamberts H, Hartman B: Psychische en sociale problemen in de huisartspraktijk: 1 . Gegevens over probleemgedrag uit het monitoringproject (Mental and social problems in general practice: 1. Data on problem behaviour from the monitoring project). Huisarts en Wetenschap 25:333-342, 1982

5. Rooijmans HGM: De verwevenheid van somatische en psychische ziekten: II. Psychiatrische stoornissen bij lichamelijk zieke patiënten (The inter-relationship of somatic and mental diseases: If. Psychiatric disturbances with physically ill patients). Ned T. voor Geneeskunde 127:2327-2331, 1983

6. Creed F, Guthrie E: Psychological treatments of the irritable bowel syndrome: a review. Gut 30:16011609,1989

7. Kroenke K, Mangelsdorff D: Common symptoms in ambulatory care: incidence, evaluation, therapy, and outcome. Am J Med 86:262-266, 1989

8. Frymoyer JW, Cats-Baril WL: An overview of the incidences and costs of low back pain. Orthop Clin North Am 22(2):263-271, 1991 
9. Deyo RA, Tsui-Wu YJ: Descriptive epidemiology of low back pain and its related medical care in the United States. Spine 12:264-268, 1987

10. Lamberts $\mathrm{H}$ : In het huis van de huisarts: verslag van het Transitieproject (In the Home of the General Practitioner: Report of the Transition Project). Lelystad: Meditekst, 1991

11. Deyo RA, Loeser JD, Bigos SJ: Herniated lumbar intervertebral disk. Ann Intern Med 112(8):598-603, 1990

12. Skovron ML: Epidemiology of low back pain. Baillieres Clin Rheumatol 6(3):559-573, 1992

13. SIG Health Care Information: Extractions from the National Medical Registration concerning admissions, hospital days and operations in 1988. Utrecht, 1990

14. GMD Common Medical Services: Numbers of Disabled Persons per Ultimo 1988 by Diagnosis, Age and Sex. Amsterdam, 1990

15. Haldeman S: Presidential address, North American Spine Society: failure of the pathology model to predict back pain. Spine 15:718-724, 1990

16. Frymoyer JW: Back pain and sciatica. $N$ Eng J Med 318:291-300, 1988

17. Waddell G: Biopsychosocial analysis of low back pain. Baillieres Clin Rheumatol 6(3):523-557, 1992

18. Polatin PB, Kinney RK, Gatchel RJ, Lillo E, Mayer TG: Psychiatric illness and chronic low-back pain. The mind and the spine-which goes first? Spine 18(1): 66-71, 1993

19. Love AW, Peck CL: The MMPI and psychological factors in chronic low back pain: a review. Pain 28:1-12, 1987

20. Manning WG, Wells KB: The effects of psychological distress and psychological well-being on use of medical services. Med Care 30:541-553, 1992

21. Saravay SM, Strain JJ: Review of outcome studies: introduction. Psychosomatics 35:227-232, 1994

22. McGill JC, Lawlis GF, Selby D, Mooney V, McCoy CE: The relationship of Minnesota Multiphasic Personality Inventory (MMPI) profile clusters to pain behaviors. J Behav Med 6:77-92, 1983

23. Battie MC, Bigos SJ: Industrial back pain complaints. A broader perspective. Orthop Clin North Am 22(2): 273-282, 1991

24. Keel PJ: Psychosocial criteria for patients selection: review of studies and concepts for understanding chronic back pain. Neurosurgery 15:935-941, 1984

25. Katon W, Gonzales J: A review of randomized trials of psychiatric consultation liaison studies in primary care. Psychosomatics 35:268-278, 1994

26. Wells KB: Depression in general medical settings: implications of three health policy studies for consultation-liaison psychiatry. Psychosomatics 35:279-296, 1994

27. Smith GR: The course of somatization and its effects on health care utilization. Psychosomatics 35:263267,1994

28. Huyse FJ, Hengeveld MW: Development of consul- tation-liaison psychiatry in the Netherlands: its social psychiatric heritage. Gen Hosp Psychiatry 11:9-15, 1989

29. Meeuwesen L, Huyse FJ, Koopmans GT, Heimans JJ: Could C/L Intervention in Low-Back Pain patients be effective. A randomized clinical trial (in press)

30. Huyse FJ, Strain JJ, Hengeveld MW: Interventions in consultation-liaison psychiatry: the development of a scheme and a checklist for operationalized interventions. Gen Hosp Psychiatry 10:88-101, 1988

31. American Psychiatric Association: Diagnostic and Statistical Mannual of Mental Disorders (3rd ed, revised). American Psychiatric Association, Washington DC, 1987

32. Hemert van AM, Hengeveld MW, Metz ME: Verwevenheid somatische en psychische stoornissen: Onderzoek protokol (Medical and psychiatric comorbidity: research protocol). University Hospital, Leiden, 1988

33. Goldberg D, Williams P: A User's Guide to the General Health Questionnaire. Windsor, Berkshire, UK, NFER-NELSON, 1988

34. Koeter MWJ, Ormel J: De Nederlandse handleiding voor de General Health Questionnaire (The Dutch Manual of the General Health Questionnaire). Lisse, Swets \& Zeitlinger BV, 1991

35. Arrindell WA, Ettema JHM. SCL-90: Handleiding bij een multidimensionele en psychopathologieindicator (SCL-90: Manual of a Multi-dimensional Psychopathology Indicator). Lisse, Swets Test Services, 1986

36. Meeuwesen L, Arrindell WA, Huyse FJ: Psychometrische kwaliteiten van de Symptom Checklist (SCL90) bij poliklinische patienten met buikpijn of lage rugklachten (Psychometric qualities of the symptom checklist (SCL-90) in medical outpatients with abdominal pain of low back pain). T voor Sociale Gezondheidszorg 70:123-131, 1992

37. Aaronson NK, Bullinger M, Ahmedzai S: A modular approach to quality-of-life assessment in cancer trials. Recent Results Cancer Res 111:231-249, 1988

38. Cohen J: Statistical Power Analysis for the Behavioral Sciences, 2nd ed. Hillsdale, NJ, Lawrence Erlbaum, 1988

39. Altmaier EM, Lehmann TR, Russel DW, Weinstein JN, Kao CF: The effectiveness of psychological interventions for the rehabilitation of low back pain: a randomized controlled trial evaluation. Pain 490(3): 329-335, 1992

40. Guthrie E, Creed F, Dawson D, Tomenson B: A controlled trial of psychological treatment for the irritable bowel syndrome. Gastroenterology 100:450-57, 1991

41. Verhaak PFM, Collijn D, Wijkel D, vd Feltz-Cornelis C, Huyse FJ. Psychiatrisch consult in de huisartsen praktijk; Somatiserende patienten. [Psychiatric consultation in primary care practice; somatizing patients]. Medisch Contact 49:1319-1322, 1994 